\title{
A newly-identified spectral reflectance signature near the lunar South pole and the South Pole-Aitken Basin
}

\author{
Faith Vilas $^{1 *}$, Elizabeth A. Jensen ${ }^{2}$, Deborah L. Domingue ${ }^{3}$, Lucy A. McFadden ${ }^{4}$, \\ Cassandra J. Runyon ${ }^{5}$, and Wendell W. Mendell ${ }^{6}$ \\ ${ }^{1}$ NASA Johnson Space Center/KR, Houston, Texas 77058 \\ ${ }^{2}$ Center for Astrophysics and Space Sciences, University of California, San Diego, La Jolla, California \\ ${ }^{3}$ Applied Physics Laboratory, Johns Hopkins Road, Laurel, Maryland 20723 \\ ${ }^{4}$ University of Maryland, Department of Astronomy, College Park, Maryland 20742 \\ ${ }^{5}$ Department of Geology, College of Charleston, Charleston, South Carolina 29424 \\ ${ }^{6}$ NASA Johnson Space Center/KA, Houston, Texas 77058
}

(Received January 12, 2007; Revised June 17, 2007; Accepted October 6, 2007; Online published February 12, 2008)

\begin{abstract}
Signal analysis of Galileo images of the Moon suggests the presence of an absorption band centered near $0.7 \mu \mathrm{m}$ in the reflectance spectra of areas located adjacent to the equatorward walls of lunar craters at latitudes ranging from -58 to $-78^{\circ}$, and areas contained in the South Pole-Aitken Basin. We propose three potential explanations: an $\mathrm{Fe}^{2+} \rightarrow \mathrm{Fe}^{3+}$ charge transfer transition in oxidized iron in clinopyroxenes (high-Ca bearing pyroxenes) or phyllosilicates (Fe- and $\mathrm{Mg}$-bearing sheet silicates containing adsorbed $\mathrm{H}_{2} \mathrm{O}$ and interlayer $\mathrm{OH}^{-}$), or an effect of titanium in ilmenite (a common lunar opaque material). No identification of the mineralogy is conclusive. The presence and nature of the absorption feature could be confirmed using AMICA images of the lunar far side from the Japanese mission Hayabusa, spectroscopic results from the Japanese mission Selene scheduled for launch in 2007, or the Moon Mineralology Mapper on the Indian mission Chandrayaan-1.
\end{abstract}

Key words: Moon, lunar surface composition, spectral reflectance, lunar mineralogy, lunar remote sensing.

\section{Motivation and Background for Search for} Lunar Phyllosilicates

Spectral observations of reflected sunlight from the surface of a planetary regolith serve as a remote sensing probe of surface mineralogical composition and particle state. Variations from the solar spectrum in the spectrum of a planetary regolith, apparent in the form of broad absorption features, reflect the presence of electronic charge transfers and vibrations between ions governed by structural spacing within crystals of a specific mineralogy (c.f., Burns, 1993). By identifying and analyzing these spectral variations, the underlying mineralogy of the planetary regolith can be determined. The identification of different minerals provides clues to the processes that formed or altered these solar system bodies. On the Moon, regolith particles of diameters $\leq 25 \mu \mathrm{m}$ dominate the remotely-sensed spectral properties of the surface over the visible/near-infrared wavelength region (Pieters et al., 1993a).

Water ice has long been postulated to exist in permanently shaded areas at the lunar poles (c.f., Watson et al., 1961; Arnold, 1979). Recent observations provide conflicting evidence for the presence and form of lunar polar water ice. Clementine bistatic radar detected a weak sig-

*Present address: MMT Observatory, P.O. Box 210065, University of Arizona, Tucson, Arizona 85721.

Copyright (c) The Society of Geomagnetism and Earth, Planetary and Space Sciences (SGEPSS); The Seismological Society of Japan; The Volcanological Society of Japan; The Geodetic Society of Japan; The Japanese Society for Planetary Sciences; TERRAPUB nal at the lunar South pole attributed to water ice (Nozette et al., 1996), while Lunar Prospector has recently detected large amounts of $\mathrm{H}$ at both poles (Feldman et al., 2000, 2001). Conversely, ground-based radar searches show no bright signal suggesting water ice at either pole (Stacy et al., 1997), and Campbell et al. (2006) find no evidence of thick deposits of ice at the South pole. Reanalysis of the Clementine radar data by Simpson and Tyler (1999) suggest that the radar reflection results were caused by underlying variations in terrain. Galileo near-infrared spectra detected no sign of the prominent 3.0- $\mu \mathrm{m}$ absorption feature due to adsorbed and interlayer $\mathrm{H}_{2} \mathrm{O}$ in phyllosilicates along a swath 30-km wide in the North polar region (Kieffer, 1995). The directed impact of the Lunar Prospector into a permanentlyshadowed crater at the lunar South pole did not produce the expected water release (Isbell et al., 1999).

We undertook a search for the presence of water-bearing minerals near the lunar South pole resulting from the process of aqueous alteration. Our motivation for this study was that-if successful — this search could provide additional backing for the existence of $\mathrm{H}_{2} \mathrm{O}$ at or near the lunar poles at some time in lunar history.

In this study, we used Galileo Solid State Imager (SSI) images taken through the GRN (central wavelength = $560 \mathrm{~nm}$, width $=60 \mathrm{~nm}), \operatorname{RED}(670 \mathrm{~nm}, 60 \mathrm{~nm}), 756 \mathrm{~nm}$ $(18 \mathrm{~nm})$, and $889 \mathrm{~nm}(120 \mathrm{~nm})$ broadband filters to search for spectral evidence of aqueous alteration near the lunar South pole by detecting signs of a broad $(\sim 260 \mathrm{~nm}$ wide) yet weak (expected absorption of 2-5\%) absorption feature in the lunar reflectance spectrum centered near $0.7 \mu \mathrm{m}$. 
This feature, attributed to an $\mathrm{Fe}^{2+} \rightarrow \mathrm{Fe}^{3+}$ charge transfer transition in six-fold coordination in oxidized iron in phyllosilicates, is present in reflectance spectra of many terrestrial phyllosilicates, CM2 carbonaceous chondrite meteorites, and many low-albedo asteroids (c.f., Vilas and Gaffey, 1989; Vilas et al., 1994; King and Clark, 1989). The $0.7-\mu \mathrm{m}$ feature is not, however, unique to iron-bearing phyllosilicates. It also appears in the reflectance spectra of high-calcium iron-bearing terrestrial clinopyroxenes (c.f., Cloutis and Gaffey, 1993). An absorption feature in laboratory reflectance spectra of some lunar samples containing titanium-bearing ilmenite also has very similar spectral characteristics (c.f., Pieters and Taylor, 1989; Tompkins and Pieters, 1999). This proposed titanium feature is not well understood (c.f., Pieters and Taylor, 1989; Tompkins and Pieters, 1999). All three of these minerals could be present in the lunar surface material; none of these minerals dominates the lunar surface material in the mineralogical state that produces the absorption feature near $0.7 \mu \mathrm{m}$. In our search for lunar phyllosilicates, we anticipated that phyllosilicates would be located in surface regolith near to locations on the moon where we expected water would survive long enough for the action of aqueous alteration to occur. We therefore expected to see the $0.7-\mu \mathrm{m}$ absorption feature signature near the lunar poles, and not near the lunar equator. We report the results of our search in this paper.

Prior to the Lunar Prospector mission, evidence from Clementine bistatic radar (Nozette et al., 1996) suggested that ice is present at the South pole. Therefore, we initially focused on this region. Lunmap 14 comprises a sequence of whole-disk images from the Galileo Earth-Moon pass 1 (EM1) taken through the seven filters of the Galileo SSI (c.f., Gaddis et al.). Gaddis et al. (1995) recalibrated images taken from six of the seven filters, describing in detail the reprocessing of these images, including "(1) incorporation of upgraded calibration files from the Jet Propulsion Laboratory (JPL) into radiometric calibration procedures, (2) subpixel coregistration, (3) application of a scattered light removal technique, (4) geometric control and reprojection, (5) photometric-function normalization, and (6) calibration to Earth-based spectral data." They used Hapke photometric-function parameters derived for diskintegrated lunar observations by Helfenstein and Veverka (1987), and empirically adapted these parameters for the Galileo EM1 data. These are therefore the most rigorously calibrated images covering terrain near the lunar South pole that we can use to delineate the $0.7-\mu \mathrm{m}$ absorption feature in spectral space (Gaddis et al., 1995). These images form the basis of the analysis in our paper. We note that the trajectory of the Galileo Earth-Moon pass 1 limited the observable lunar latitudes to those below $\sim+60^{\circ}$, so that a similar analysis near the lunar North pole could not be conducted with Lunmap 14 or other images from EM1.

\section{Data Analysis}

The transmission curves of the four Galileo filters (obtained from T. Hiroi, personal communication) were modeled using a technique described by Vilas et al. (1997) where piecewise fits to the transmission response at different wavelengths are created across the passbands of the

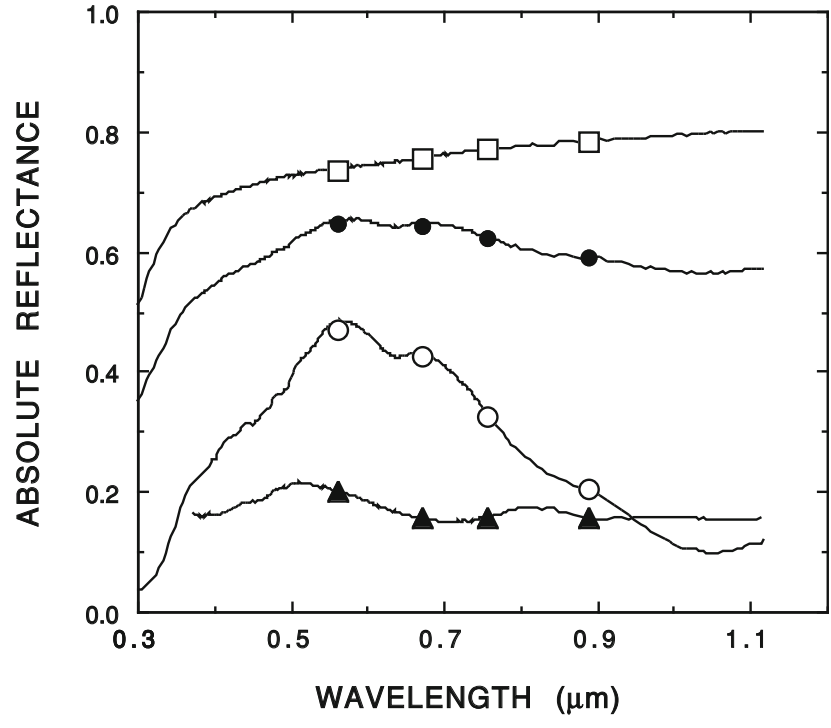

Fig. 1. Sample absolute reflectance spectra, and synthetic Galileo filter values made from these spectra, of four materials (top to bottom) (a) anorthosite [plagioclase feldspar: $(\mathrm{Ca}, \mathrm{Na})(\mathrm{Al}, \mathrm{Si}) \mathrm{AlSi}_{2} \mathrm{O}_{8}$ ] (open squares), (b) $33 \%$ olivine- $67 \%$ anorthosite combination (closed circles), (c) olivine $\left[(\mathrm{Mg}, \mathrm{Fe})_{2} \mathrm{SiO}_{4}\right]$ (open circles), and (d) antigorite $[(\mathrm{Mg}$, $\left.\left.\mathrm{Al}, \mathrm{Fe})_{6}(\mathrm{Si}, \mathrm{Al})_{4} \mathrm{O}_{10}(\mathrm{OH})_{8}\right)\right]$ (closed triangles). Only the antigorite-a phyllosilicate - correctly shows an absorption feature that could be attributed to the $0.7-\mu \mathrm{m}$ absorption. Note that identification of the $0.7-\mu \mathrm{m}$ feature accommodates significant variations in overall spectral slope. Reflectance spectra have a dispersion of $50 \AA$ per element. Spectra are from the USGS mineral library (Clark et al., 2003) and King and Clark (1989).

four individual filters. For each Galileo filter, synthetic Galileo photometry was then calculated for ground-based reflectance spectra of asteroids and laboratory reflectance spectra of meteorite and terrestrial rock samples, both with and without the $0.7-\mu \mathrm{m}$ absorption feature. This was done by combining the individual points of the higher spectral resolution reflectance spectrum of the desired object (e.g., asteroid, laboratory sample) with the modeled Galileo filter transmission (Fig. 1). This process allows us to demonstrate how these higher spectral resolution reflectance properties of Solar System objects or laboratory mineral samples would appear through the Galileo broadband filters. We can then compare them with Galileo lunar photometry (Gaddis et al., 1995). As seen in these synthetic reflectance spectra, the $0.7-\mu \mathrm{m}$ absorption feature is weak ( 2 to $5 \%$ depth from the background continuum). When a spectrum containing the $0.7-\mu \mathrm{m}$ feature is coupled with the "reddened" lunar spectrum (reflectance increasing with increasing wavelength), the $0.7-\mu \mathrm{m}$ feature might not be identified using only a simple ratio of images in two different filters. This is because the much greater spectral slope could mask the weaker absorption feature, if the background continuum is not removed (see Vilas, 1994, for a discussion of this issue).

In the selected areas of Lunmap 14 outlined in Fig. 2, the images were first visually aligned to each other, pixel by pixel, in order to correct the slight changes in Galileo's position between image acquisitions. Counts for each pixel from all four Galileo images were then smoothed using a 3-pixel $\times 3$-pixel running box average to remove any noise effects at these scales. We next converted these pixel 


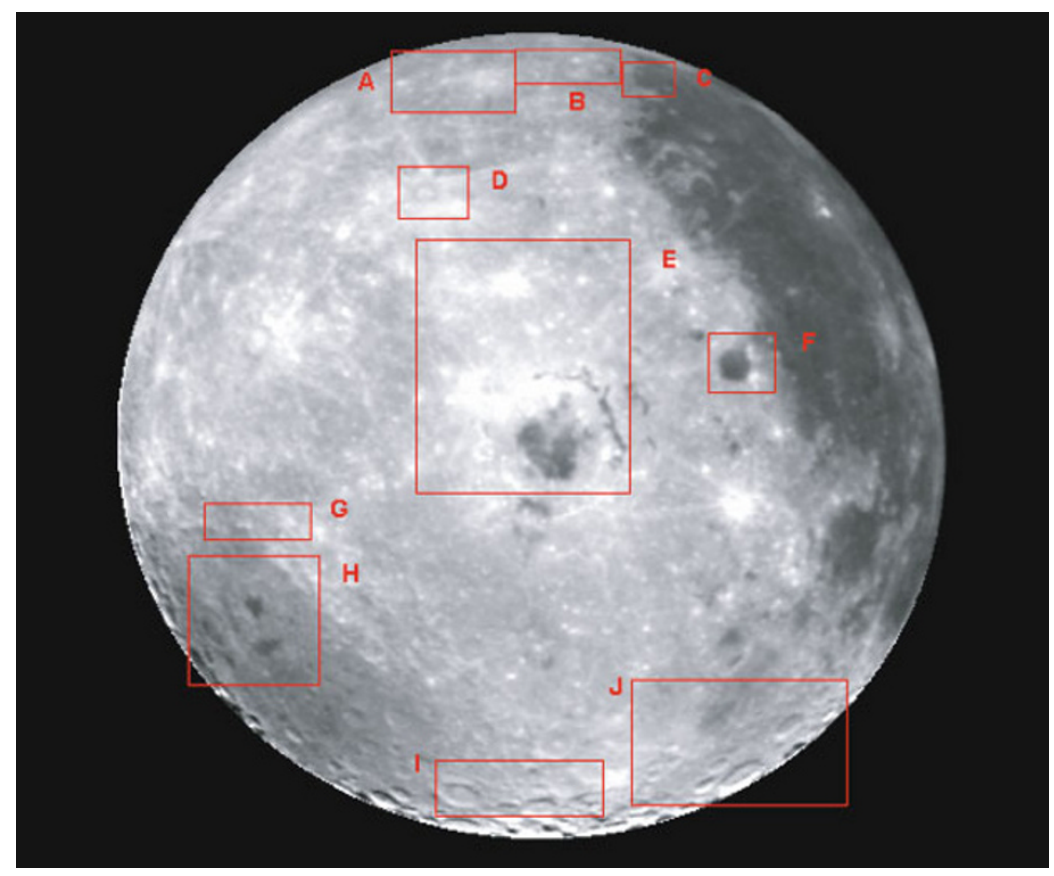

Fig. 2. The Lunmap 14 GRN (560 nm) image with areas that were searched for the $0.7-\mu \mathrm{m}$ feature in Galileo photometry outlined (Gaddis et al., 1995). Strips near the edge of the image were excluded from this study where we felt that the fidelity of the photometry was insufficient. The spatial resolution is $8 \mathrm{~km} /$ pixel, sub-spacecraft coordinates are latitude $=-20.7^{\circ}$, longitude $=98.3^{\circ}$, with a $20^{\circ}$ phase angle (Gaddis $e t$ al., 1995 ).

counts to Moon/Sun reflectance values for each filter using the conversion factors listed in table I of Gaddis et al. (1995). Finally, for each pixel location, the four Galileo filter values were scaled to 1.0 at $560 \mathrm{~nm}(\mathrm{GRN})$. The relative reflectance values were then examined. The presence or absence of the absorption feature near $0.7 \mu \mathrm{m}$ would be manifested by a negative inflection in the value of the RED filter compared to the linear background defined by the GRN and 756 filter values (c.f., Fig. 1). The presence of two other spectral attributes that dominate the spectral reflectance properties across the Moon can also be identified in the photometry from these four Galileo filters. First, the lower-wavelength side of the M1 mafic silicate absorption feature, indicating the presence of iron-bearing pyroxenes or olivines or both, would be manifested by a decrease in the 889 filter value relative to the 756 filter value (c.f., Clark, 1957; Burns, 1993; Adams and McCord, 1972). Second, the presence and magnitude of a reddened slope in a lunar spectrum indicate the effects of the creation of nanophase iron from micrometeoroid impact, solar wind impact, or energetic cosmic and solar rays (c.f., Pieters et al., 2000; Hapke, 2001; Allen et al., 1993). This broadband photometry does not have sufficient spectral resolution for detailed study of the underlying mineralogy. In Fig. 1, we plot the absolute reflectance spectra of four sample materials and their synthetic Galileo filter values, in order to demonstrate how these materials would appear if their reflectance properties were measured using these broadband filters.

The majority of Lunmap 14 pixels show the spectral signatures expected for lunar regolith showing spectral reddening combined with the effects of the $\mathrm{Fe}^{2+}$ absorption at $0.9-1.0 \mu \mathrm{m}$ in pyroxene. We report here the identification of concentrations of pixels adjacent to the north (equatorward) walls of large, degraded craters with absorptions
Table 1. Craters with phyllosilicates on adjacent walls.

\begin{tabular}{c|c|cc}
\hline Marker & Crater $^{1}$ & Longitude $\left(^{\circ}\right)$ & Latitude $\left(^{\circ}\right)$ \\
\hline $\mathrm{a}$ & Gruemberger & $10 \mathrm{~W}$ & -66.9 \\
$\mathrm{~b}$ & Klaproth & $26 \mathrm{~W}$ & -69.8 \\
$\mathrm{c}$ & Blancanus & $21.4 \mathrm{~W}$ & -63.8 \\
$\mathrm{~d}$ & Clavius & $15 \mathrm{~W}$ & -49 \\
$\mathrm{e}$ & Drygalski & $84.9 \mathrm{~W}$ & -79.3 \\
$\mathrm{f}$ & Zeeman & $133.6 \mathrm{~W}$ & -75.2 \\
$\mathrm{~g}$ & Ashbrook & $112.5 \mathrm{~W}$ & -81.4 \\
$\mathrm{~h}$ & Rocky terrain ENE of Drygalski & est. $65 \mathrm{~W}$ & est. -76 \\
$\mathrm{i}$ & Unnamed crater N. of Boltzmann & $75.7 \mathrm{~W}$ & -74.6 \\
$\mathrm{j}$ & De Forest & $162.1 \mathrm{~W}$ & est. -77.3 \\
\hline
\end{tabular}

${ }^{1}$ Crater identification (where known) and central coordinates from USGS tabulation in the NASA Planetary Data System.

suggesting a center near $0.7 \mu \mathrm{m}$ superimposed on the typical Moon/Sun spectrum at latitudes ranging from $\sim-58^{\circ}$ to $-78^{\circ}$ (Table 1, Fig. 3). We note that some pixels in the South Pole-Aitken (SPA) Basin region also have signatures suggesting the $0.7-\mu \mathrm{m}$ absorption feature (Fig. 3). Closer to the equator, the SPA regions with $0.7-\mu \mathrm{m}$ absorption features do not correlate with walls of large craters.

\section{Testing the Veracity of the Absorption Feature Signature}

We tested the registration among the images in all four filters in order to determine if the detection of the absorption feature was a function of potential misregistration of the images. In a remotely-sensed image of an atmosphereless planetary body such as the Moon, the underlying topography contributing to the signal in each pixel can cause large albedo variations between adjacent pixels. For the areas of Lunmap 14 that we sampled as part of this study 


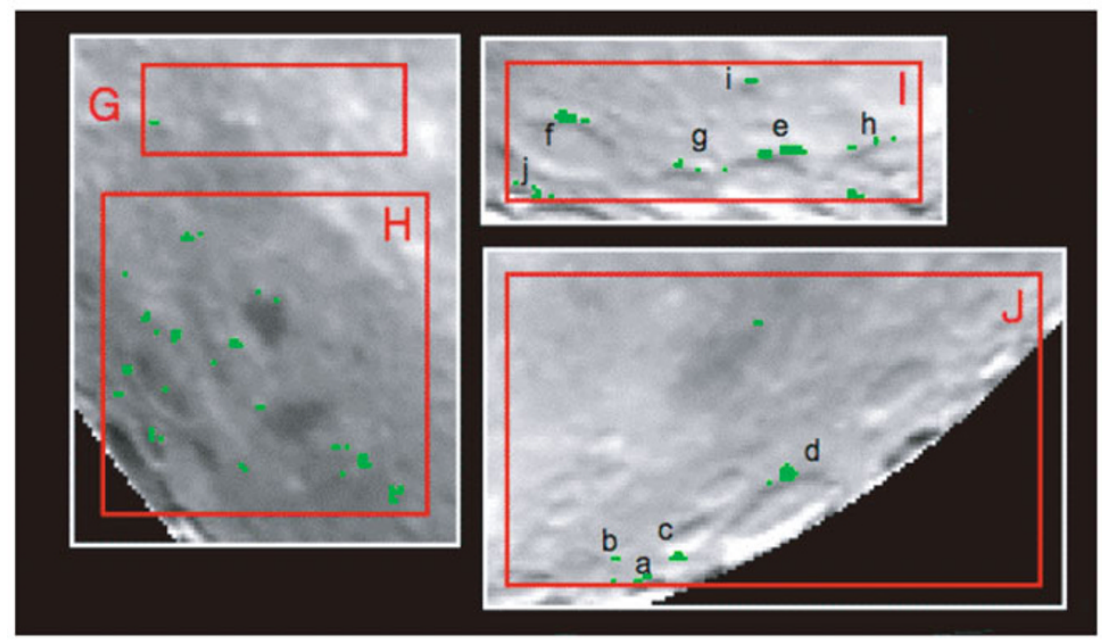

Fig. 3. Lunmap 14 pixels testing positively for the presence of a $0.7-\mu \mathrm{m}$ feature are colored in green in regions G, H, I, J. These subsets shown are from the Lunmap 14 GRN image. The spatial resolution is $8 \mathrm{~km} /$ pixel (Gaddis et al., 1995). Locations where the images test positively for the 0.7- $\mu \mathrm{m}$ feature have been marked with alphabetical letters that are listed alongside the crater tabulation in Table 1.

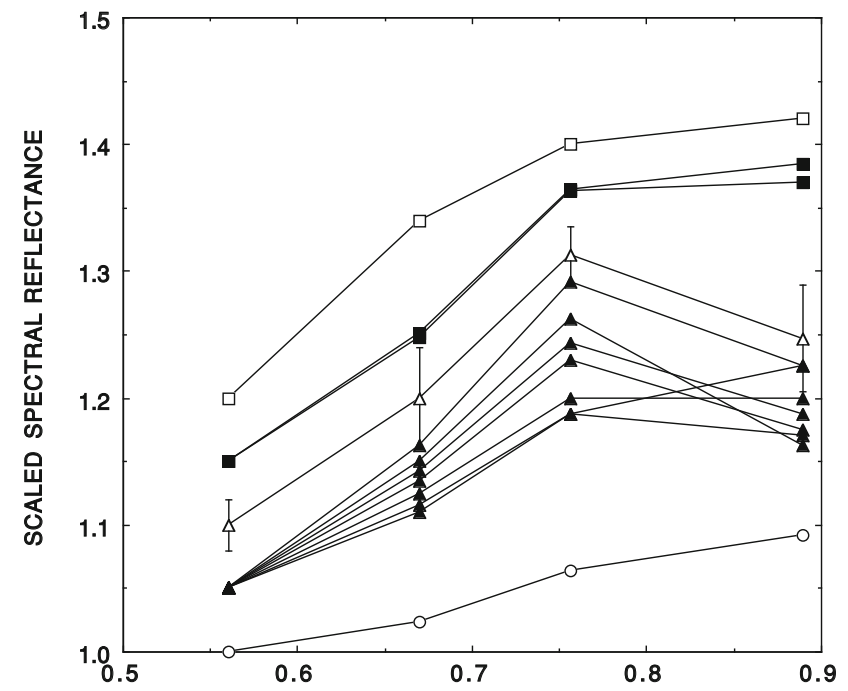

WAVELENGTH $(\mu \mathrm{m})$

Fig. 4. Scaled reflectance photometry showing the presence and absence of the $0.7-\mu \mathrm{m}$ feature represented in Galileo filters: dark triangles are samples from near South pole region I with feature; dark squares are samples from South Pole-Aitken Basin region G with feature; open squares are a sample of lunar photometry from area $\mathbf{J}$ without feature (the most common reflectance signature across Lunmap 14); open circles are result of linear mixing model with $33 \%$ antigorite $[(\mathrm{Mg}, \mathrm{Al}$, $\left.\left.\mathrm{Fe})_{6}(\mathrm{Si}, \mathrm{Al})_{4} \mathrm{O}_{10}(\mathrm{OH})_{8}\right)\right]$ particles (particle size $>250 \mu \mathrm{m}$ ) (King and Clark, 1989), and $67 \%$ Galileo lunar photometry from region J not containing the $0.7-\mu \mathrm{m}$ absorption feature. Error bars shown for one sample of Galileo photometry (open triangles) from region I are the standard deviation of the statistical mean for the 9 pixels measured in the running box average. A more detailed discussion of SSI data errors is presented in Pieters et al. (1993b). All photometry is scaled to 1.0 for the GRN filter. Different photometric groups discussed here are offset by a factor of 0.05 in reflectance for clarity.

(Fig. 2), our first check was to ratio the images in each filter to images in each other filter, as a quick look to identify any possible misregistration. If the images are correctly registered, variations in intensity across the image will not show large albedo variations between adjacent pixels. Only the 889-filter image in region I (closest to the lunar South pole) showed apparent misalignment to the region I images taken through the other three filters. Since the intensity of values acquired through the 889 filter affects the identification of the presence and strength of the M1 absorption feature, but not the identification of the $0.7-\mu \mathrm{m}$ feature, this will not affect our determination of the $0.7-\mu \mathrm{m}$ feature in region I. Second, the veracity of the possible $0.7-\mu \mathrm{m}$ absorption was tested by shifting pixels in one filter image up, down, left, or right by one pixel with respect to the other three images. Shifting images in the GRN, 756, or 889 filters did not change the pixel locations of any identified $0.7-\mu \mathrm{m}$ absorptions. In contrast, shifting the RED filter image caused pixel locations showing $0.7-\mu \mathrm{m}$ absorption features to shift one pixel in the same direction that the RED filter image was shifted.

This shows that the cause of the absorption tracks with the Galileo filter value that would reflect the presence of an absorption feature having the width and central wavelength of the $0.7-\mu \mathrm{m}$ absorption feature. Therefore, the absorption feature is real and not an artifact of registration. Examples of lunar Galileo photometry comparing the presence and absence of this absorption feature are shown in Fig. 4.

\section{Identification and Location of Regions Showing the Proposed 0.7- $\mu \mathrm{m}$ Feature}

The areas outlined on Lunmap 14 (Fig. 2) were searched in order to sample different areas of the lunar surface for similar spectral signatures showing the presence of the 0.7$\mu \mathrm{m}$ feature. Among the four northern hemisphere regions (A, B , C , D) sampled, we examined large-diameter craters, and terrains of contrasting albedo as seen in these four Galileo filters. These areas include maria. Near the equator, a large area including Mare Orientale (E) was selected. Region E covers terrains of widely contrasting albedo. Here, areas were sampled where the surface temperatures are too great to allow water to remain on the surface long enough to allow aqueous alteration to proceed. Our intention here was 


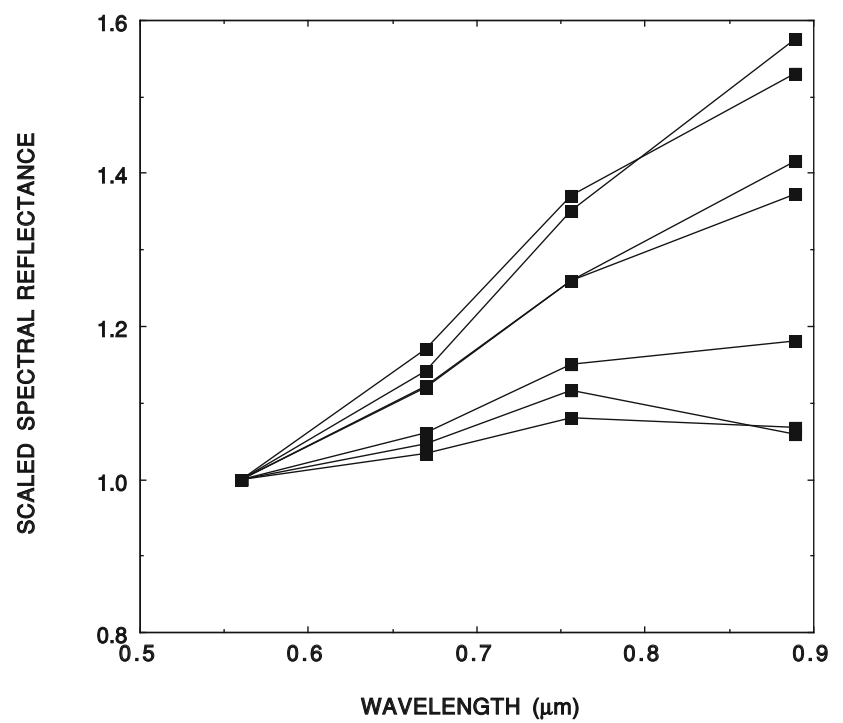

Fig. 5. Scaled synthetic Galileo filter values of seven samples of lunar melts containing ilmenite that have reflectance spectra showing an absorption feature near 0.6-0.7 $\mu \mathrm{m}$ (Tompkins et al., 1997). All photometry is scaled to 1.0 for the GRN filter. Reflectance spectra have a dispersion of $50 \AA$ per element.

to determine if we would identify the presence of the 0.7 $\mu \mathrm{m}$ feature in terrain where we had no possible expectation of aqueous alteration; such an identification would rule out the presence of phyllosilicates as a cause of the absorption feature. An area encompassing Grimaldi was also sampled (F), in order to include a region of contrasting albedo that has been well studied by earth-based telescopic measurements (c.f., Hawke et al., 1991). None of the lunar regions tested in the northern hemisphere (A, B, C, D) or around the equator (E, F) showed any sign of an absorption feature near $0.7 \mu \mathrm{m}$.

Region I was chosen to approach the South pole as closely as feasible. Areas of the Moon covering parts of the South Pole-Aitken Basin and adjacent lunar highlands terrain $(\mathrm{G}, \mathrm{H})$ close to the lunar limb and at varying distances from region I were sampled. An area located at southern latitudes near the Moon's limb comprising large, complex lunar craters ( $\mathrm{J}$ ) was also tested. Areas adjacent to the north (equatorward) walls of some large, complex craters in regions $\mathrm{I}$ and $\mathrm{J}$ showed signatures indicating an absorption feature near $0.7 \mu \mathrm{m}$. The portions of these regions closest to the limb were not considered in order to avoid data with large photometric variations across the pixels.

Areas of the Moon covering parts of the South PoleAitken Basin and adjacent lunar highlands terrain $(\mathrm{G}, \mathrm{H})$ close to the lunar limb were sampled. Here, we found pixels in the South Pole-Aitken Basin region $(\mathrm{G}, \mathrm{H})$ that also indicate an absorption feature centered near $0.7 \mu \mathrm{m}$ (Fig. 4). Closer to the equator, regions $\mathrm{G}$ and $\mathrm{H}$ do not correlate with walls of large craters. The FeO content of the SPA appears greater than the surrounding areas, as evidenced by analyses of Clementine photometry suggesting that orthopyroxenes and some olivines are present in the SPA region (c.f., Lucey et al., 1998). The identification of pixels having this spectral feature in the SPA suggests that the cause of this (a)

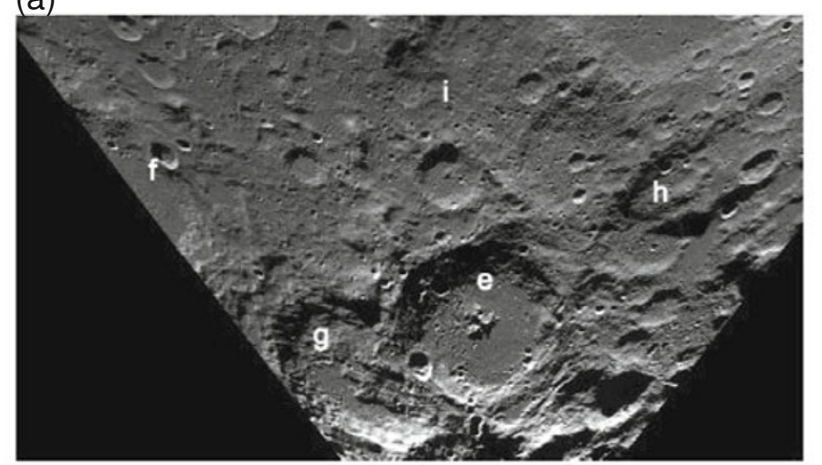

(b)

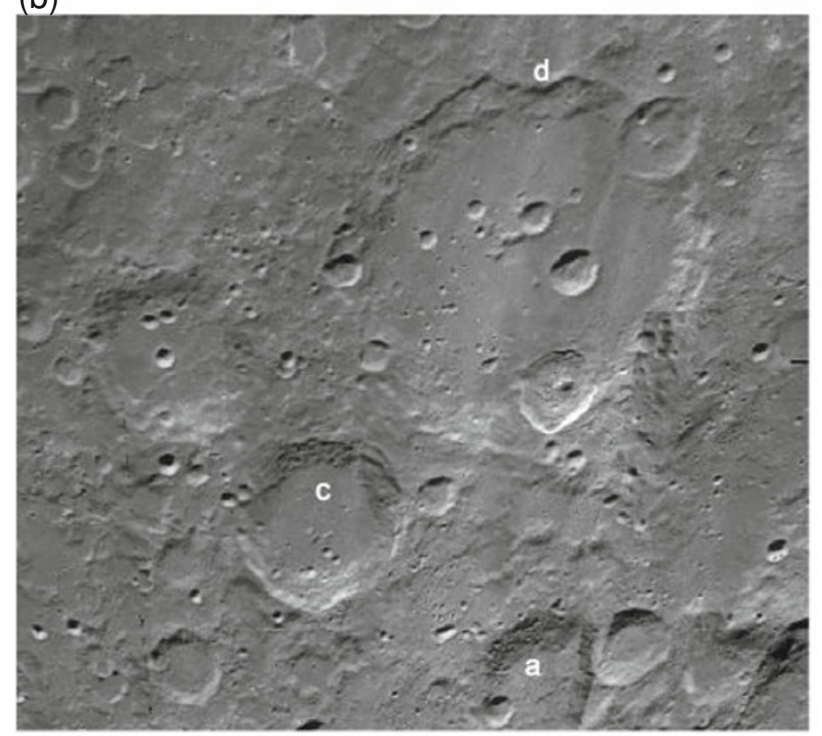

Fig. 6. Clementine images of some regions near the lunar South pole where the $0.7-\mu \mathrm{m}$ absorption feature has been identified in Galileo imagery. (a) Partial coverage of region I centered on crater Drygalski. (b) Partial coverage of region J centered on crater Clavius. Locations where the images test positively for the $0.7-\mu \mathrm{m}$ have been marked with alphabetical letters that are listed alongside the crater tabulation in Table 1.

absorption feature might not be phyllosilicates.

We explored the detection method to determine the minimum percentage of the $\mathrm{Fe}^{2+} \rightarrow \mathrm{Fe}^{3+}$ absorption feature necessary to produce a spectral signal suggesting the absorption at $0.7 \mu \mathrm{m}$. We used a linear mixing model to combine Galileo reflectance values of highlands terrain near the larger craters in regions I and $\mathrm{J}$ with synthetic Galileo photometry made from the available laboratory spectrum of a terrestrial antigorite (a phyllosilicate) sample (King and Clark, 1989). A spatial unit represented by a pixel containing 25 to $33 \%$ antigorite produces the RED filter inflection we found near the lunar South pole. Below $25 \%$, the antigorite signature is not strong enough to be spectrally apparent. Above 33\%, other signatures rarely if ever found in the Galileo data dominate the mixing samples.

The Clementine spacecraft obtained images of the Moon at much higher spatial resolution, but in VNIR filters of 415 , $750,900,950,1000 \mathrm{~nm}$. These filters are not spectrally distributed to be able to show the presence of the $0.7-\mu \mathrm{m}$ feature, however, the images provide a more detailed view of the underlying topography in the regions where we see 
the $0.7-\mu \mathrm{m}$ feature in the Lunmap 14 image. Near the lunar South pole, these regions all consist of rough, hilly terrain separate from the equatorward walls of large craters (examples are shown in Fig. 6), representing areas where the surface material appears to have been churned through previous surface event or events. The extreme illumination angles at which the inflection in the RED filter was observed in the Galileo photometry motivated a study by Domingue and Vilas (2006) to consider the effects of underlying lunar surface topography on photometry. The purpose of this study was to address whether extreme lighting conditions on the lunar surface (as existed near the lunar South pole when the Lunmap 14 images were acquired by Galileo) could artificially cause absorption features in lunar reflectance spectra. While the depth of existing absorption features and strength of apparent slope of VNIR reflectance spectra can be altered by the extreme illumination angles, no absorption feature was artificially created by the effects of underlying topography (Domingue and Vilas, 2006). Thus, the inflection in the RED filter is not an artifact of photometric effects caused by unresolved lunar topography.

\section{Possible Origins for the Observed Absorption Feature}

We propose here three possible explanations for the origin of the RED filter absorption feature in lunar regolith. None is a perfect candidate for the underlying mineralogy that would produce this absorption feature. We do not favor any specific explanation in this paper.

\subsection{Clinopyroxenes}

Ferric iron $\left(\mathrm{Fe}^{3+}\right)$ in terrestrial clinopyroxenes produces an absorption feature centered near $0.6-0.7 \mu \mathrm{m}$ due to an $\mathrm{Fe}^{2+} \rightarrow \mathrm{Fe}^{3+}$ charge transfer transition in six-fold coordination in oxidized iron (Cloutis and Gaffey, 1991, 1993), whose presence and depth are not necessarily tied to the characteristics of the $\mathrm{Fe}^{2+}$ absorption feature centered near $1 \mu \mathrm{m}$ (Cloutis and Gaffey, 1993). Although clinopyroxene is a major component of the lunar soil, the returned lunar samples are very reduced with no apparent $\mathrm{Fe}^{3+}$ (c.f., Taylor, 1981; Heiken et al., 1991). In order to create the $\mathrm{Fe}^{3+}$ in the more oxidized clinopyroxenes necessary to produce this absorption feature, the lunar rock material would need to be completely melted. Terrestrial clinopyroxenes require heating to temperatures $>1100^{\circ} \mathrm{C}$ before recrystallization, in order to create highly oxidized clinopyroxenes (Deer et al., 1966). This requires a lunar thermal event that would produce temperatures high enough to melt rock in these extremely localized places on the lunar surface where we observe evidence of this absorption feature. A smaller, localized event is difficult to envision. Material that has been created in a larger lunar geological (likely, impact), and which was subsequently buried, could have become partially exposed through surface erosion or gardening processes over time.

\subsection{Ilmenite}

Pieters and Taylor (1989) observe an absorption feature that is centered near $0.6 \mu \mathrm{m}$ of uncertain origin in spectra of some lunar clasts. They suspected that the origin of the feature was submicroscopic ilmenite, although they could not confirm the identification. Tompkins et al. (1997) ob- serve absorption features centered near $0.6 \mu \mathrm{m}$ in laboratory reflectance spectra of lunar impact melts. Tompkins et al. (1997) also attribute these absorption features to finegrained ilmenite, and note that the strength of this absorption feature could depend more strongly on grain shape and size than on mineral abundance. Neither Pieters and Taylor (1989), nor Tompkins et al. (1997), propose a specific charge transfer or crystal field transition to explain the absorption. We created synthetic Galileo filter photometry for the reflectance spectra of seven lunar impact melt samples measured by Tompkins et al. (1997), and the inflection in the RED filter is present for all seven samples (Vilas et al., 1999) (Fig. 5). Thus, the preservation of ilmenite mixed with glasses as observed by Tompkins et al. (1997) around impact craters is a viable possibility for the origin of these absorption features. We looked for the $0.7-\mu \mathrm{m}$ absorption feature signature in and near young craters with bright surrounding material present in the Lunmap 14 images: the signature that we observed near the lunar South pole is not observed near the craters closer to the lunar equator.

\subsection{Phyllosilicates}

Near the lunar South pole, we see the RED filter signature in rough terrain located adjacent to older, complex craters in regions I and J. An explanation for the $0.7-\mu \mathrm{m}$ spectral signature on the equatorward sides of these craters was offered by Vilas et al. (1999). We do not believe that this explanation is sufficient for the detections of the spectral signatures in areas of the SPA, nor necessarily correct for the $0.7-\mu \mathrm{m}$ signatures near the lunar South pole. Water is delivered to the lunar polar regions through solar wind reduction of iron in the lunar regolith (c.f. Housley et al., 1973, 1974; Allen et al., 1993), or comet or water-bearing meteor impact (c.f., Gibson and Moore, 1973), or both. The hydrogen is implanted in the lunar soil and reacts with the iron oxide in minerals and glasses $\left(\mathrm{FeO}+\mathrm{H}_{2} \rightarrow \mathrm{Fe}+\mathrm{H}_{2} \mathrm{O}\right)$, creating minor amounts of water vapor and iron metal (Housley et al., 1973, 1974). The Fe metal produced serves as a darkening agent for the lunar regolith. Across most of the Moon, especially nearer the equator, the water vapor created by these events would sublime quickly from the surface due to the high surface temperatures (although 5$10 \%$ could migrate to any permanently shadowed regions of the Moon at the poles (see the explanation of mechanism by Hunten et al., 1988)). At or near the lunar poles, theoretical studies suggest that temperatures in the permanently shadowed regions of craters would be low enough to retain water ice across the lifetime of the Moon (Hodges, 1980). Larger, flat-shaped complex craters with more vertical crater walls would also generate lower internal temperatures than simple, bowl-shaped craters due to differences in the internal radiometry generated by the crater structure (Hodges, 1980; Paige et al., 1992; Ingersoll et al., 1992; Butler et al., 1993). Hence, the equatorward (cooler, sometimes shadowed) walls of the large, complex craters located near the poles could retain water vapor, liquid or ice over longer time scales. A lunar surface temperature of $100 \mathrm{~K}$ not in shadow will retain water for $\sim 100 \mathrm{yr} ; 50 \mathrm{~K}$ will retain water for billions of years (Hodges, 1980). At latitudes of $\pm 60^{\circ}-80^{\circ}$ on the Moon, temperatures $\leq 100 \mathrm{~K}$ would be expected in shaded portions of larger craters (Hodges, 1980). 
On any planetary surface that is a vacuum (asteroids, the moon), ice or liquid water will be subject to losses from sublimation or Lyman $\alpha$ radiation and sputtering (c.f., Arnold, 1979; Lanzerotti et al., 1986; Morgan and Shemansky, 1991), if it is not buried. On the moon, burial by regolith gardening would trap the ice or liquid water. Once buried, the aqueous alteration process creating phyllosilicates can occur freely. The time scales for aqueous alteration reactions vary, requiring hours to years, depending on temperatures, compositions, and water:rock ratios of the starting materials. We propose that over the lifetime of these older craters, buried phyllosilicates in small quantities have been produced at the equatorward walls of the craters and, through gardening due to continuous micrometeoroid impact, laterally mixed with the anhydrous silicate surface material near the surface rim, and exposed over the lunar lifetime. The adsorbed water in phyllosilicates could be lost to Lyman $\alpha$ radiation, however, the interlayer water will be retained (M. Zolensky, personal communication). The evidence from reflectance observations of aqueously-altered asteroids (c.f., Rivkin et al., 2002) shows that dessication from the lack of a protective atmosphere would not affect phyllosilicates on the lunar surface.

In an extensive laboratory study, Cocks et al. (2002) have demonstrated that adsorbed water on extremely finegrained $(0.05 \mu \mathrm{m})$ gamma alumina $\left(\mathrm{Al}_{2} \mathrm{O}_{3}\right)$ powder (used as a proxy for ilmenite), and goethite $(\mathrm{FeO}-\mathrm{OH})$ powder (an iron oxide), buried below the lunar surface, can produce hydrated minerals and adsorbed water stable over the lunar geologic lifetime. Cocks et al. (2002) suggest that this mechanism could explain the $\mathrm{H}$ detected at the lunar North pole and part of the lunar South pole.

\section{Spacecraft Evidence for the $0.7-\mu \mathrm{m}$ Feature: Present and Future}

Independent confirmation of the presence of the $0.7-\mu \mathrm{m}$ feature is necessary. No additional Galileo data can be processed to the extent of Lunmap 14: the spacecraft ephemeris files have been accidentally lost for both Earth-Moon passes and cannot be recreated. Spectral reflectance data from present and future lunar missions can confirm (or negate) the presence of this absorption feature.

\subsection{Hayabusa and Selene missions}

The best immediate confirmation of this signature is offered by two Japanese Space Agency space probes. The Japanese mission Hayabusa used an Earth-Moon gravity assist on May 19, 2004, to reach the near-Earth asteroid 25143 Itokawa in September, 2005. The Asteroid Multiband Imaging Camera (AMICA) imaged the lunar far side. Filters chosen for the AMICA instrument were selected to match the Eight-Color Asteroid Survey (ECAS) filters. Vilas (1994) determined an algorithm to identify the $0.7-\mu \mathrm{m}$ feature in ECAS asteroid spectra. We propose that these signatures could be confirmed by the Hayabusa AMICA images of the Moon, if they covered the target areas near the lunar South pole. The Hayabusa data were made accessible to the public by JAXA in spring, 2007, and plans exist to deposit these data in the Planetary Data System.

The Japanese mission Selene is targeted to launch in July, 2007, to orbit the Moon and study its properties. Mineralog- ical composition data can be acquired by the Spectral Profiler, which will obtain spectra across the wavelength region of 0.5 to $2.6 \mu \mathrm{m}$ at a spectral resolution of 60 to $80 \mathrm{~nm}$ over a footprint size of $500 \mathrm{~m}$, and should detect this absorption feature if it is present. Coupled with the elemental compositional instruments, the nature of the hydrogen at the lunar poles should be resolved. The Multiband Imager will have 9 broadband filters spaced across the 0.4- to 1.6-nm with an increased-resolution footprint of $20 \mathrm{~m}$ (Ohtake et al., 2006), however, the spectral locations of these filters will not delineate the $0.7-\mu \mathrm{m}$ feature.

\subsection{Chandrayaan-1 mission}

The Indian space agency mission Chandrayaan-1 will carry a U.S. instrument to map lunar mineralogy (the Moon Mineralogy Mapper) across a spectral range of 430 to $3000 \mathrm{~mm}$ at a resolution of $20 \mathrm{~nm}$, and search for ice at the lunar poles (the Miniature Synthetic Aperture Radar), in addition to a European X-ray spectrometer. These instruments could confirm the presence or absence of this feature and the state of hydrogen at the lunar poles.

\section{Summary}

We searched for the spectral signature of an absorption band frequently seen in the reflectance spectra of phyllosilicates, and centered spectrally near $0.7 \mu \mathrm{m}$, in Galileo Lunmap 14 images of the moon. We detected the presence of material having this signature near the outer (equatorward) walls of lunar craters located at southern declinations, and also at locations within the South Pole-Aitken Basin. The identification of this absorption feature at locations closer to the lunar equator suggests that it is not necessarily caused by the presence of phyllosilicates. Three possible explanations for the underlying material that causes the absorption include clinopyroxenes, phyllosilicates, and fine-grained ilmenite. Japanese and Indian robotic missions scheduled to study the moon within the next two years include spectrographs that can resolve the presence and nature of this absorption feature.

Acknowledgments. We thank K. Jarvis, C. Toyama, B. Wade, and N. Fuller for help during the lifetime of this project. F. Vilas acknowledges support from the NASA Lunar Data Analysis Program. D. L. Domingue acknowledges support from the Lunar and Planetary Institute staff scientist program in addition to support from NASA's NEAR Discovery mission program. C. Runyon acknowledges support from NASA grant NAG5-4198.

\section{References}

Adams, J. B. and T. B. McCord, Electronic spectra of pyroxenes and interpretation of telescopic spectral reflectivity curves of the moon, Proc. 3rd Lunar Sci. Conf., 3021-3034, 1972.

Allen, C. C., R. V. Morris, H. V. Lauer, and D. S. McKay, Microscopic iron metal on glass and minerals - a tool for studying regolith maturity, Icarus, 104, 291, 1993.

Arnold, J., Ice in the lunar polar regions, J. Geophys. Res., 84, 5659, 1979. Burns, R. G., Mineralogical Applications of Crystal Field Theory, $2^{\text {nd }}$ Ed., Cambridge Univ. Press, New York, 1993.

Butler, B. J., D. O. Muhleman, and M. A. Slade, Mercury: Full-disk radar images and the detection and stability of ice at the North pole, J. Geophys. Res., 98, 15003, 1993.

Campbell, D. B., B. A. Campbell, L. M. Carter, J.-L. Margot, and N. J. S. Stacy, No evidence for thick deposits of ice at the lunar south pole, Nature, 433, 835, 2006.

Clark, R. N., G. A. Swayze, R. Wise, K. E. Livo, T. M. Hoefen, R. F. Kokaly, and S. J. Sutley, USGS Digital Spectral Library splib05a, USGS 
Open File Report 03-395, 2003.

Clark, S. P., Jr., Absorption spectra of some silicates in the visible and near infrared, Am. Mineral., 42, 732, 1957.

Cloutis, E. A. and M. J. Gaffey, Pyroxene spectroscopy revisited: Spectralcompositional correlations and relationship to geothermometry, J. Geophys. Res., 89, 6329, 1991.

Cloutis, E. A. and M. J. Gaffey, Lunar regolith analogues: Spectral reflectance properties of compositional variations, Icarus, 102, 203, 1993.

Cocks, F. H., P. A. Klenk, S. A. Watkins, W. N. Simmons, J. C. Cocks, E. E. Cocks, and J. C. Sussingham, Lunar ice: Adsorbed water on subsurface polar dust, Icarus, 160, 386, 2002.

Deer, W. A., R. A. Howie, and J. Zussman, An Introduction to the Rock Forming Minerals, New York, John Wiley and Sons, 1966.

Domingue, D. L. and F. Vilas, Local topographic effects on photometry and reflectance spectra of planetary surfaces: An example based on lunar photometry, Meteoritics Planet. Sci., 2006 (in press).

Feldman, W. C., S. Maurice, A. B. Binder, B. L. Barraclough, R. C. Elphic, and D. J. Lawrence, Polar hydrogen deposits on the Moon, J. Geophys. Res., 105, 4175, 2000.

Feldman, W. C., S. Maurice, D. J. Lawrence, R. C. Little, S. L. Lawson, O. Gasnault, R. C. Wiens, B. L. Barraclough, R. C. Elphic, and T. H. Prettyman, Evidence for Water Ice near the lunar poles, J. Geophys. Res., 106(E10), 23231, 2001

Gaddis, L., A. McEwen, and T. Becker, Compositional variations on the Moon: Recalibration of Galileo solid-state imaging data for the Orientale region and far side, J. Geophys. Res., 100, 26345, 1995.

Gibson, E. K. and G. W. Moore, Volatile-rich lunar soil: Evidence of possible cometary impact, Science, 179, 69, 1973.

Hapke, B., Space weathering from Mercury to the asteroid belt, J. Geophys. Res., 106, 10039, 2001.

Hawke, B. R., P. G. Lucey, G. J. Taylor, J. F. Bell, C. A. Peterson, D. T. Blewett, K. Horton, G. A. Smith, and P. D. Spudis, Remote sensing studies of the Orientale region of the moon-a pre-Galileo view, Geophys. Res. Lett., 18, 2141, 1991.

Heiken, G., D. Vaniman, and B. M. French, Lunar Sourcebook: A User's Guide to the Moon, Cambridge University Press, 1991.

Helfenstein, P. and J. Veverka, Photometric properties of lunar terrains derived from Hapke's equations, Icarus, 72, 342, 1987.

Hodges, R. R., Lunar cold traps and their influence on argon-40, Proc. Lunar Plan. Sci. Conf. 11th, 2463, 1980.

Housley, R. M., R. W. Grant, and N. E. Paton, Origin and characteristics of excess Fe metal in lunar glass welded aggregates, Proc. Lunar Sci. Conf. 4th, 2737, 1973.

Housley, R. M., E. H. Cirlin, N. E. Paton, and I. B. Goldberg, Solar wind and micrometeorite alteration of the lunar regolith, Proc. Lunar Sci. Conf. 5th, 2623, 1974.

Hunten, D. M., T. H. Morgan, and D. E. Shemansky, The Mercury atmosphere, in Mercury, edited by F. Vilas, C. R. Chapman, and M. W. Matthews, Tucson, University of Arizona Press, 562, 1988.

Ingersoll, A. P., T. Svitek, and B. C. Murray, Stability of polar frosts in spherical bowl-shaped craters on the Moon, Mercury, and Mars, Icarus, 100, 40, 1992

Isbell, D., D. Morse, and B. Rische, No Water Ice Detected on the Moon, NASA Spacelink, NASA Press Release 99-10-13, 1999.

Kieffer, H. H., A spectral search for $\mathrm{H}_{2} \mathrm{O}$ near the lunar North pole, Bul. Amer. Astro. Soc., 27, 1110, 1995.

King, T. V. V. and R. N. Clark, Spectral characteristics of chlorites and Mgserpentines using high-resolution reflectance spectroscopy, J. Geophys. Res., 94, 13997, 1989.

Lanzerotti, L. J., W. L. Brown, and R. E. Johnson, Astrophysical implications of ice sputtering, Nucl. Instrum. Methods Phys. Res., B14, 373, 1986.
Lucey, P. G., D. T. Blewett, and B. R. Hawke, FeO and TiO2 concentrations in the South Pole-Aitken Basin: Implications for mantle composition and basin formation, J. Geophys. Res., 103, 3701, 1998.

Morgan, T. H. and D. E. Shemansky, Limits to the lunar atmosphere, $J$. Geophys. Res., 96, 1351, 1991.

Nozette, S., C. L. Lichtenberg, P. Spudis, R. Bonner, W. Ort, E. Malaret, M. Robinson, and E. M. Shoemaker, The Clementine bistatic radar experiment, Science, 274, 1495, 1996.

Ohtake, M., J. Haruyama, S. Mastunaga, T. Morota, S. Kodama, and LISM team, Observations and data analysis plan of the SELENE multiband imager, Lunar Plan. Sci. XXXVII, (cd-rom), 2006.

Paige, D. A., S. E. Wood, and A. R. Vasavada, The thermal stability of water ice at the poles of Mercury, Science, 258, 643, 1992.

Pieters, C. M. and G. J. Taylor, Millimeter petrology and kilometer mineralogical exploration of the moon, Proc. $19^{\text {th }}$ Lunar Planet. Sci. Conf., 115, 1989.

Pieters, C. M., E. M. Fischer, O. Rode, and A. Basu, Optical effects of space weathering: The role of the finest fraction, J. Geophys. Res., 98 , 20817, 1993a.

Pieters, C. M., J. M. Sunshine, E. M. Fischer, S. L. Murchie, M. Belton, A. McEwen, L. Gaddis, R. Greeley, G. Neukum, R. Jaumann, and H. Hoffmann, Crustal diversity of the Moon: Compositional analyses of Galileo SSI data, J. Geophys. Res., 98, 17127, 1993b.

Pieters, C. M., L. A. Taylor, S. K. Noble, L. P. Keller, B. Hapke, R. V. Morris, C. C. Allen, D. S. McKay, and S. Wentworth, Space weathering on airless bodies: Resolving a mystery with lunar samples, Meteorit. Planet. Sci., 35, 1101, 2000.

Rivkin, A. S., E. S. Howell, F. Vilas, and L. A. Lebofsky, Hydrated minerals on asteroids: the astronomical record, in Asteroids III, edited by W. R. Bottke, A. Cellino, P. Paolicchi, and R. P. Binzel, 235, Tucson, University of Arizona Press, 2002.

Simpson, R. A. and G. L. Tyler, Reanalysis of Clementine bistatic radar data from the lunar south pole, J. Geophys. Res., 104, 3845, 1999.

Stacy, N. J. S., D. B. Campbell, and P. G. Ford, Arecibo radar mapping of the lunar poles: A search for ice deposits, Science, 276, 1527, 1997.

Taylor, S. R., Planetary Science: A Lunar Perspective, Houston, Texas: Lunar and Planetary Institute, 1981.

Tompkins, S. and C. M. Pieters, Mineralogy of the lunar crust: Results from Clementine, Meteorit. Planet. Sci., 34, 25, 1999.

Tompkins, S., C. M. Pieters, and G. Ryder, Spectral characteristics of lunar impact melts-Implications for remote sensing, LPSC XXVIII, 441, 1997.

Vilas, F., A cheaper, faster, better way to detect water of hydration on Solar System bodies, Icarus, 11, 456, 1994.

Vilas, F. and M. J. Gaffey, Phyllosilicate absorption features in main-belt and outer-belt asteroid reflectance spectra, Science, 246, 790, 1989.

Vilas, F., K. S. Jarvis, and M. J. Gaffey, Iron alteration minerals in the visible and near-infrared spectra of low-albedo asteroids, Icarus, $\mathbf{1 0 9}$, 274, 1994.

Vilas, F., E. A. Jensen, and L. A. McFadden, Extracting spectral information about 253 Mathilde using the NEAR photometry, Icarus, 129, 440, 1997.

Vilas, F., D. L. Domingue, E. A. Jensen, L. A. McFadden, C. R. Coombs, and W. W. Mendell, Aqueous alteration on the moon, Lunar Planet. Sci. XXX, (cd-rom), 1999.

Watson, K., B. C. Murray, and H. Brown, On the possible presence of ice on the Moon, J. Geophys. Res., 66, 1598, 1961.

F. Vilas (e-mail: fvilas@mmto.org), E. A. Jensen, D. L. Domingue, L. A. McFadden, C. J. Runyon, and W. W. Mendell 